\title{
Global assessment of molecularly identified Anisakis Dujardin, 1845 (Nematoda: Anisakidae) in their teleost intermediate hosts
}

\author{
Thomas Kuhn ${ }^{1}$, Frank Hailer ${ }^{2}$, Harry W. Palm ${ }^{3}$ and Sven Klimpel ${ }^{1}$ \\ ${ }^{1}$ Biodiversity and Climate Research Centre, Medical Biodiversity and Parasitology, Senckenberg Gesellschaft für Naturforschung \\ and Goethe-University, Institute for Ecology, Evolution and Diversity, Frankfurt/Main, Germany; \\ ${ }^{2}$ Biodiversity and Climate Research Centre, Evolutionary Genomics, Senckenberg Gesellschaft für Naturforschung, Frankfurt/ \\ Main, Germany; \\ ${ }^{3}$ Aquaculture and Sea-Ranching, Faculty of Agricultural and Environmental Sciences, University Rostock, Rostock, Germany
}

\begin{abstract}
Here, we present the ITS ribosomal DNA (rDNA) sequence data on 330 larvae of nematodes of the genus Anisakis Dujardin, 1845 collected from 26 different bony fish species from 21 sampling locations and different climatic zones. New host records are provided for Anisakis simplex (Rudolphi, 1809) sensu stricto (s.s.) and A. pegreffii Campana-Rouget et Biocca, 1955 from Anoplopoma fimbria (Pallas) (Santa Barbara, East Pacific), A. typica (Diesing, 1860) from Caesio cuning (Bloch), Lepturacanthus savala (Cuvier) and Katsuwonus pelamis (Linnaeus) (Indonesia, West Pacific), A. simplex s.s. from Cololabis saira (Brevoort) (Hawaii, Central Pacific), A. simplex C of Nascetti et al. (1986) from Sebastolobus alascanus Bean (Santa Barbara, East Pacific) and A. physeteris Baylis, 1923 from Synaphobranchus kaupii Johnson (Namibia, East Atlantic). Comparison with host records from 60 previous molecular studies of Anisakis species reveals the teleost host range so far recorded for the genus. Perciform (57 species) and gadiform (21) fishes were the most frequently infected orders, followed by pleuronectiforms (15) and scorpaeniforms (15). Most commonly infected fish families were Scombridae (12), Gadidae (10), Carangidae (8) and Clupeidae (7), with Merluccius merluccius (Linnaeus) alone harbouring eight Anisakis species. Different intermediate host compositions implicate differing life cycles for the so far molecularly identified Anisakis sibling species.
\end{abstract}

Keywords: Internal transcribed spacer (ITS), life cycle ecology, toothed/baleen whales, anisakiasis, paratenic host, sibling species, host range

Anisakid nematodes have a worldwide distribution and are known as causative agents of the human anisakidosis, a painful inflammation of the gastrointestinal tract caused by the ingestion of the third-stage larvae $\left(\mathrm{L}_{3}\right)$. Infections in humans can be acquired by ingestion of raw or insufficiently cooked fish and fisheries products (Sakanari and McKerrow 1989, Nadler et al. 2005, Audicana and Kennedy 2008). Due to the high prevalence in commercially important fish species such as Clupea harengus Linnaeus or species of Oncorhynchus Suckley, members of the genus Anisakis Dujardin, 1845 can be considered the most common causative agents of this zoonosis (disease: anisakiasis) (Mattiucci and Nascetti 2008, Hochberg and Hamer 2010, Karl et al. 2011). Besides the major clinical symptoms such as vomiting, fever or epigastric pain, the incidence of Anisakis-related severe allergic disorders is a frequently documented problem and reflects the necessity of continuous research efforts in this field (Daschner and Pascual 2005, Audicana and Kennedy 2008, Daschner et al. 2011).
Anisakis has an indirect, heteroxenous life cycle including four larval stages. Typical definitive hosts are toothed and baleen whales (Cetacea) of the families Delphinidae, Ziphiidae, Physeteridae and Kogiidae, but species of the Balaenopteridae, Pontoporidae, Monodontidae, Phocoenidae, Neobalaenidae and even Otariidae are known to become infected (e.g. Klimpel et al. 2004, 2010a, Nadler et al. 2005, Mattiucci and Nascetti 2008, Kuhn et al. 2011). The eggs are shed into to the surrounding water column with the faeces of the definitive hosts. The larvae hatch and are subsequently ingested by small crustaceans (Køie et al. 1995, Marcogliese 1995, Køie 2001, Busch et al. 2012). They are then transferred within the food chain into larger macroinvertebrates (e.g. amphipods, cephalopods), where they grow in length and become infective to fishes and definitive hosts (Klimpel et al. 2004, 2008, McClelland 2005, Mattiucci and Nascetti 2008, Busch et al. 2012). Macroinvertebrates as well as small schooling fish are thought to be important intermediate/paratenic

Address for correspondence: T. Kuhn, Biodiversity and Climate Research Centre, Medical Biodiversity and Parasitology, Senckenberg Gesellschaft für Naturforschung, Goethe-University, Institute for Ecology, Evolution and Diversity, Senckenberganlage 25, D-60325 Frankfurt/Main, Germany. Phone: +49 697542 1896; Fax: +49 697542 7904; E-mail: thomas.kuhn@senckenberg.de 
hosts. Anisakis is also capable of infecting larger piscivorous hosts (e.g. Gadus morhua Linnaeus, Merluccius merluccius [Linnaeus]) without further moult. This leads to the accumulation of enormous numbers of larvae and enhances the transmission probability to their respective definitive hosts (Lile 1998, Hammerschmidt et al. 2009, Klimpel and Palm 2011, Busch et al. 2012).

Until molecular techniques became routine diagnostic tools, the identification of larval anisakid nematodes was difficult and largely based on slight differences in the arrangements and morphometrics of the internal structures such as the alimentary tract, the orientation of the excretory pore and the shape of the tail (Mattiucci and Nascetti 2008, Klimpel and Palm 2011). In the case of the morphological almost indistinguishable species Anisakis (cryptic species), a correct assignment to their respective species was not feasible, which often led to erroneous identifications (Klimpel and Palm 2011). Population-based multilocus enzyme-electrophoresis (MAE) as well as PCR-based approaches such as PCR-RFLP (Restriction Fragment Length Polymorphism), SSCP (Single-Strand Conformation Polymorphism) or DNA sequencing of genetic markers have remarkably increased our knowledge of the actual species diversity and species composition (e.g. Anderson et al. 1998, Klimpel et al. 2008). Application of these methods to taxonomic studies of Anisakis spp. has revealed the existence of nine distinct species with different host preferences, ecology and zoogeography (e.g. Klimpel et al. 2004, 2008, Valentini et al. 2006, Mattiucci and Nascetti 2008).

The genus comprises two major clades; the first clade includes the $A$. simplex-complex (A. simplex [Rudolphi, 1809] sensu stricto [s.s.], A. pegreffii Campana-Rouget et Biocca, 1955, A. simplex C of Nascetti, Paggi, Orecchia, Smith, Mattiucci et Bullini, 1986) as well as A. typica (Diesing, 1860) and two sister-species A. nascettii Mattiucci, Paoletti et Webb, 2009 and A. ziphidarum Paggi, Nascetti, Webb, Mattiucci, Cianchi et Bullini, 1998. The second clade consists exclusively of the A. physeteris-complex (A. brevispiculata Dollfus, 1966, A paggiae Mattiucci, Nascetti, Dailey, Webb, Barros, Cianchi et Bullini, 2005, A. physeteris [Baylis, 1923]) (Mattiucci et al. 2009, Klimpel et al. 2011, Kuhn et al. 2011).

Because of the abundance of anisakids in commercially important fishes and its implication for human health, an enormous number of host records of Anisakis has accumulated in the literature, however, often resulting only from morphological identification. Klimpel and Palm (2011) suggested that not all Anisakis species are dangerous to humans, and thus a closer look to the real host range of the different species is necessary. So far, no comprehensive overview has illustrated the host and distribution patterns of Anisakis spp., making it difficult to uncover the life cycle ecology and zoogeographical distribution patterns.
The present study summarizes a large sample size of Anisakis individuals from different teleost intermediate hosts, geographical regions and climate zones, using direct sequencing of the ITS molecular marker. The obtained data set is combined with already existing parasite-host records based on molecular identification, demonstrating the different hosts and zoogeographical distribution patterns for the species. Implications for the life cycles of nine different Anisakis species are discussed.

\section{MATERIALS AND METHODS}

\section{Sample collection}

A total of 330 Anisakis third-stage larvae were isolated from 26 teleost species from 21 localities around the world (Table 1; in part Kuhn et al. 2011). Specimens were extensively washed in $0.9 \%$ saline solution and identified morphologically to the genus level according to Anderson (2000). All samples were preserved in $\mathrm{EtOH}(100 \%)$ prior to molecular examination.

\section{Molecular analysis}

Genomic DNA was isolated and purified from individual larvae using a genomic DNA extraction kit (Peqlab Biotechnology $\mathrm{GmbH}$, Erlangen) according to the instructions of the manufacturer. The rDNA region comprising the ITS-1, 5.8S, ITS-2 and flanking sequences (= ITS + ) was amplified using the primers NC2 (5'-TTA-GTT-TCT-TTT-CCT-CCG-CT-3') and TK1 (5'-GGC-AAA-AGT-CGT-AAC-AAG-GT-3') (Zhu et al. 2000, Kuhn et al. 2011). PCR-reaction $(50 \mu l)$ included $25 \mu$ Master-Mix (Peqlab Biotechnology $\mathrm{GmbH}$, Erlangen) containing dNTP, $\mathrm{MgCl}_{2}$, Buffer and Taq-Polymerase, $3 \mu \mathrm{l}$ of each primer $(10 \mathrm{pmol} / \mu \mathrm{l}), 14 \mathrm{ddH}_{2} \mathrm{O}$ and $5 \mu \mathrm{l}$ genomic DNA extract.

PCR reactions were performed in an Advanced Primus 96 thermocycler (Peqlab) under the following conditions: initial denaturation at $95^{\circ} \mathrm{C}$ for $1 \mathrm{~min} ; 40$ cycles of $94^{\circ} \mathrm{C}$ for $45 \mathrm{sec}$ (denaturation), $55^{\circ} \mathrm{C}$ for $45 \mathrm{sec}$ (annealing), $72^{\circ} \mathrm{C}$ for $45 \mathrm{sec}$ (extension); final extension at $72^{\circ} \mathrm{C}$ for $10 \mathrm{~min}$. Negative controls (reactions without DNA) were included in each PCR run. PCR products were examined on $1 \%$ agarose gels and purified with Cycle-Pure Kit (Peqlab Biotechnology GmbH, Erlangen) and a total volume of $7 \mu \mathrm{l}$, including $2 \mu \mathrm{l}$ primer $(100 \mathrm{pmol} / \mu \mathrm{l})$ and $5 \mu \mathrm{l}$ of the PCR product ( $250 \mathrm{ng} / \mu \mathrm{l})$ was sequenced by Seqlab (Goettingen $\mathrm{GmbH}$ ). Both spacers and the $5.8 \mathrm{~S}$ gene from each PCR product were sequenced using the primer TK1.

\section{Sequence analyses}

For sequence analyses, electropherograms were checked manually and sequences were aligned using CLUSTALX (v.2.0.1.0) (Larkin et al. 2007) to visualize genetic polymorphisms. For species identification, sequence data of each reaction were compared with previously published data in GenBank by using the BLASTn algorithm (Altschul et al. 1990).

\section{RESULTS AND DISCUSSION}

The ITS ribosomal DNA (rDNA) sequence data of 330 larvae of Anisakis spp. from 26 different bony fishes and 21 localities revealed the presence of five out of nine currently recognized Anisakis species, namely A. simplex s.s., A. pegreffii, A. simplex C, A. typica, A. physeteris, and two genotypes (Anisakis sp. SAN2004, Ani- 
Table 1. Information on the nematodes identified by ITS sequence analyses. Sampling localities, host and Anisakis species are provided for 330 nematodes.

\begin{tabular}{|c|c|c|c|c|c|c|c|c|c|c|c|}
\hline Sample location & Host & $\begin{array}{l}\text { D } \\
\text { 章 } \\
\text { 至 }\end{array}$ & $\begin{array}{l}00 \\
\stackrel{0}{<}\end{array}$ & $\underset{\Xi}{\circlearrowright}$ & 龸 & $\frac{\lambda}{\stackrel{2}{\alpha}}$ & 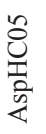 & 龸 & 黾 & Total & Accession-No. \\
\hline \multirow[t]{2}{*}{ Alaska (Bay Nushagak) } & Oncorhynchus keta & 12 & - & - & - & - & - & - & - & 12 & JN968655-JN968666 \\
\hline & Oncorhynchus nerka & 11 & - & - & - & - & - & - & - & 11 & JN968667-JN968677 \\
\hline \multirow[t]{2}{*}{ Alaska (Cordova) } & Oncorhynchus keta & 12 & - & - & - & - & - & - & - & 12 & JN968678-JN968689 \\
\hline & Oncorhynchus nerka & 12 & - & - & - & - & - & - & - & 12 & JN968690-JN968701 \\
\hline Antarctic (South Shetland Islands) & Gymnoscopelus nicholsi & - & - & 14 & - & - & - & - & - & 14 & JN968638-JN968651 \\
\hline \multirow[t]{2}{*}{ Baltic Sea (Kattegat) } & Scomber scombrus & 1 & - & - & - & - & - & - & - & 1 & JN968759 \\
\hline & Clupea harengus & 11 & 1 & - & - & - & - & - & - & 12 & JN968748-JN968759 \\
\hline Baltic Sea (off Kiel) & Clupea harengus & 9 & - & - & - & - & - & - & - & 9 & JN968760-JN968768 \\
\hline Baltic Sea (off Rügen) & Clupea harengus & - & - & - & - & - & - & - & 1 & 1 & KC121366 \\
\hline \multirow[t]{2}{*}{ Barents Sea (Bellsund Banks) } & Melanogrammus aeglefinus & 13 & - & - & - & - & - & - & - & 13 & JN968770-JN968782 \\
\hline & Micromesistius poutassou & 11 & - & - & - & - & - & - & 1 & 12 & $\begin{array}{l}\text { JN968783-JN968793; } \\
\text { KC121367 }\end{array}$ \\
\hline Barents Sea (Björnöya) & Gadus morhua & 2 & - & - & - & - & - & - & - & 2 & JN968794-JN968795 \\
\hline Barents Sea (Forland Banks) & Micromesistius poutassou & 9 & - & - & - & - & - & - & - & 9 & JN968796-JN968804 \\
\hline Barents Sea (Sjubre Banks) & Melanogrammus aeglefinus & 12 & - & - & - & - & - & - & - & 12 & JN968805-JN968816 \\
\hline \multirow[t]{4}{*}{ China } & Scomberomorus niphonius & - & 3 & - & - & - & - & - & - & 3 & JN968607-JN968609 \\
\hline & Cleisthenes herzensteinii & - & 7 & - & - & - & - & - & - & 7 & JN968594-JN968600 \\
\hline & Gadus macrocephalus & - & 6 & - & - & - & - & - & - & 6 & JN968601-JN968606 \\
\hline & Trichiurus lepturus & - & 11 & - & - & - & - & - & - & 11 & JN968610-JN968620 \\
\hline \multirow[t]{3}{*}{ Hawaii } & Cololabis saira & 5 & - & - & - & - & - & - & - & 5 & JN968817-JN968821 \\
\hline & Selar crumenophtalmus & - & - & - & 6 & - & - & - & - & 6 & JN968907-JN968912 \\
\hline & Katsuwonus pelamis & - & - & - & 1 & - & - & - & - & 1 & JN968906 \\
\hline \multirow[t]{5}{*}{ Indonesia } & Trichiurus lepturus & - & - & - & 26 & - & - & - & - & 26 & JN968936-JN968961 \\
\hline & Auxis thazard thazard & - & - & - & 5 & - & - & - & - & 5 & JN968927-JN968931 \\
\hline & Auxis rochei rochei & - & - & - & 14 & - & - & - & - & 14 & JN968913-JN968926 \\
\hline & Lepturacanthus savala & - & - & - & 2 & - & 1 & - & - & 3 & $\begin{array}{l}\text { JN968934; JN968935; } \\
\text { KC121379 }\end{array}$ \\
\hline & Caesio cuning & - & - & - & 2 & - & - & - & - & 2 & JN968932, JN968933 \\
\hline Italy (Ionian Sea) & Merluccius merluccius & - & 10 & - & - & - & - & - & - & 10 & JN968621-JN968630 \\
\hline Italy (Tyrrhenian Sea) & Scomber japonicus & - & 3 & - & - & - & - & - & - & 3 & JN968631-JN968633 \\
\hline Madeira & Aphanopus carbo & 16 & - & - & - & - & - & - & - & 16 & JN968822-JN968837 \\
\hline Namibia & Synaphobranchus kaupii & - & - & - & - & 1 & - & - & - & 1 & JN968637 \\
\hline \multirow[t]{2}{*}{ Moorea } & Katsuwonus pelamis & - & - & - & 3 & - & - & - & - & 3 & JN968962-JN968964 \\
\hline & Selar crumenophtalmus & - & - & - & 1 & - & - & - & - & 1 & JN968965 \\
\hline \multirow[t]{2}{*}{ North Sea (off Scheveningen) } & Trachurus trachurus & 8 & - & - & - & - & - & - & - & 8 & JN968898-JN968905 \\
\hline & Scomber scombrus & 20 & - & - & - & - & - & - & - & 20 & JN968878-JN968897 \\
\hline North Sea (off German Coast) & Clupea harengus & 40 & - & - & - & - & - & - & 2 & 42 & $\begin{array}{l}\text { JN968838-JN968877; } \\
\text { KC121368; KC121369 }\end{array}$ \\
\hline \multirow[t]{3}{*}{ Santa Barbara } & Anoplopoma fimbria & 1 & - & 2 & - & - & - & - & - & 3 & $\begin{array}{l}\text { JN968769; JN968652; } \\
\text { JN968653 }\end{array}$ \\
\hline & Sebastolobus alascanus & - & - & 1 & - & - & - & 1 & - & 2 & JN968654; KC121371 \\
\hline & Total & 205 & 41 & 17 & 60 & 1 & 1 & 1 & 4 & 330 & \\
\hline
\end{tabular}

Asimss - Anisakis simplex s.s.; Apeg - A. pegreffi; AsimC - A. simplex C; Atyp - A. typica; AspHC05 - Anisakis sp. HC2005; AspSAN04 - Anisakis sp. SAN 2004; Aindet - Anisakis indetermined.

sakis sp. HC2005) previously recorded by Nadler et al. (2005) and Kijewska et al. (2009), respectively (Table 1). Findings of $A$. simplex s.s. and A. pegreffii in Anoplopoma fimbria (Pallas) from Santa Barbara, A. typica in Caesio cuning (Bloch), Lepturacanthus savala (Cuvier) and Katsuwonus pelamis (Linnaeus) from Indonesia and Moorea, A. simplex s.s. in Cololabis saira (Brevoort) from Hawaii, A. simplex $\mathrm{C}$ in Sebastolobus alascanus Bean from Santa Barbara as well as A. physeteris in Synaphobranchus kaupii Johnson from Namibia represent new host records (Table 1).
Most abundant was $A$. simplex s.s. with a total of 205 records, followed by A. typica (60), A. pegreffii (41), A. simplex $\mathrm{C}$ (17) and $A$. physeteris (1) (Table 1$)$. In addition, four sequences that cannot be unambiguously classified by sequence analyses were identified (Anisakis indet NSG_Char_39.5 and Anisakis indet_NSG_Char_40.8 in Clupea harengus from Scheveningen, North Sea, Anisakis indet_BalRu_Char_121.1 in C. harengus from off Rügen, Baltic Sea and Anisakis indet_BSBB_Mpou_79_8 in Micromesistius poutassou [Risso] from Belsund Bank, Barents Sea). BLAST analysis revealed the same score 
(E-value $=0.00)$ and $99.0 \%$ identity to several different sequences, $A$. simplex s.s., A. pegreffii and $A$. simplex C, respectively (Table 1 ).

The sequence Aindet_BalRu_Char_121.1 was heterozygote at positions 281 and 296 of the aligned sequences. These positions are known as diagnostic sites of the ITS marker between the sister species A. simplex s.s. and $A$. pegreffii. Abollo et al. (2003) identified hybrids between both species in a sympatric area along the Galician coast of Spain (Atlantic Ocean). The authors eliminated the regular polymorphisms as well as incomplete concerted evolution within a multicopy gene as possible reasons and cited the exchange of genetic material between both species as the most likely cause for such hybridization events. Despite limited sample size, this is further corroborated by the fact that both presumably admixed genotypes were encountered in populations where both pure species occur.

It is not known whether hybridization in Anisakis enables adaptation to particular environmental conditions or whether it is a consequence of incomplete barriers to hybrid mating, but it could reflect the radiation within the genus Anisakis. However, since DNA turnover mechanisms, including slippage during DNA replication, gene conversion, unequal crossing-over or transposition events or simply intraspecific variations cannot be excluded as cause for the polymorphisms, the hybrid hypothesis within the genus Anisakis needs to be rigorously tested and critically evaluated by analyzing natural populations and large sample sizes, and more genomic loci (Hailer et al. 2012).

The obtained sequence data were combined with the host records from 60 previous molecular studies to evaluate the role of the teleost intermediate and paratenic hosts in the life cycle of these parasites. A total of 155 teleost fish species from 71 families and 19 orders have been reported in the literature (Table 2). Perciform (57 species) and gadiform (21) fishes were the most frequently infected orders, followed by pleuronectiforms (15) and scorpaeniforms (15). The Scombridae (12), Gadidae (10), Carangidae (8), Clupeidae (7), Pleuronectidae (6) and Salmonidae (5) were the most frequently represented families, with Merluccius merluccius alone harbouring eight different Anisakis species (Table 2).

The vast majority are food fishes of high commercial interest, reflecting the extensive scientific effort that has dealt with the role of Anisakis as causative agent of the food-borne parasitic zoonosis called anisakiasis. Anisakis is usually associated with the traditions of consuming raw or insufficiently cooked fish (e.g. salted herring, sushi, sashimi) and the increasingly popular habit not to overcook food (Audicana and Kennedy 2008).

The fish hosts recorded were classified into three habitats (pelagic, benthopelagic, demersal), according to the definitions provided by Froese and Pauly (2012). Pelagic fish species live and feed in the open sea and are usually associated with the surface or the middle depths of a water body. Benthopelagic fishes are living near the bottom as well as in midwaters, where they feed on benthic and/ or free swimming organisms. In contrast, demersal fishes live and feed on or near to the sea floor (Froese and Pauly 2012). Our data demonstrate that 71 of the 155 reported hosts live in pelagic and benthopelagic environments.

Nearly the same number (72) is demersal and another 12 are known to be reef-associated (Froese and Pauly 2012) (Fig. 1). Although the majority of fishes have been classified demersal, many of them are known to extend their feeding range into the benthopelagic and feed on both benthic and (bentho-)pelagic organisms (e.g. species of Merluccius, Gadus Linnaeus, Pollachius Nilsson, Hippoglossus Cuvier and Conger Bosc). Taking into account the large number of hosts of each species that live in pelagic, demersal and benthopelagic habitats, it becomes apparent that the life cycle of Anisakis includes food chains throughout the water column with a bias towards (bentho-)pelagic environments (see also Fig. 1).

Anisakis pegreffii (101 records) was the most frequently reported species in the literature, followed by $A$. simplex s.s. (63) (Table 2). This is very likely due to the fact that the former species is widely distributed in western Pacific waters around Japan, where most food-borne diseases like anisakiasis occur (e.g. Klimpel and Palm 2011, Kuhn et al 2011). However, there was no consensus whether A. pegreffii and $A$. simplex s.s. complete life cycles mainly in pelagic or in benthic habitats (e.g. Mattiucci et al. 1997, Abollo et al. 2001, Mattiucci and Nascetti 2008). Abollo et al. (2001) demonstrated that $80 \%$ of the fish hosts of A. simplex s.s. are nektobenthic or demersal, whereas no infection with $A$. pegreffi was found in large pelagic cephalopods. Our data demonstrate that both parasite species as well as $A$. simplex $\mathrm{C}$ have similar proportions of fish hosts that live in pelagic, benthopelagic and demersal habitats suggesting similar life cycle strategies (Fig. 1).

These data are congruent with the so far known spectrum of definitive hosts reported for these species. The very closely related $A$. simplex s.s., $A$. pegreffii and A. simplex $\mathrm{C}$ mature mainly in oceanic dolphins, which are known to feed offshore on large pelagic and benthopelagic fishes (Højgaard 1998, Jefferson et al. 2008, Klimpel et al. 2011, Kuhn et al. 2011). This would suggest a mainly (bentho-) pelagic life cycle for these species. According to Klimpel et al. (2004, 2007), it seems that at least $A$. simplex s.s. can perform a pelagic life cycle in the Norwegian Deep off the continental shelf.

With 26 records of $A$. typica, 16 of $A$. physeteris, 7 of A. ziphidarum, 6 of A. nascettii and A. paggiae and 5 of $A$. brevispiculata, respectively, these species were far less frequently reported from fish intermediate hosts. However, considering the feeding behaviour of the reported cetacean definitive hosts, e.g. Ziphius cavirostris Cuvier, Phy- 
Table 2. Teleost intermediate/paratenic hosts for Anisakis spp. so far detected by molecular analyses. Order, family, host, habitat, ocean and references are given for each Anisakis species.

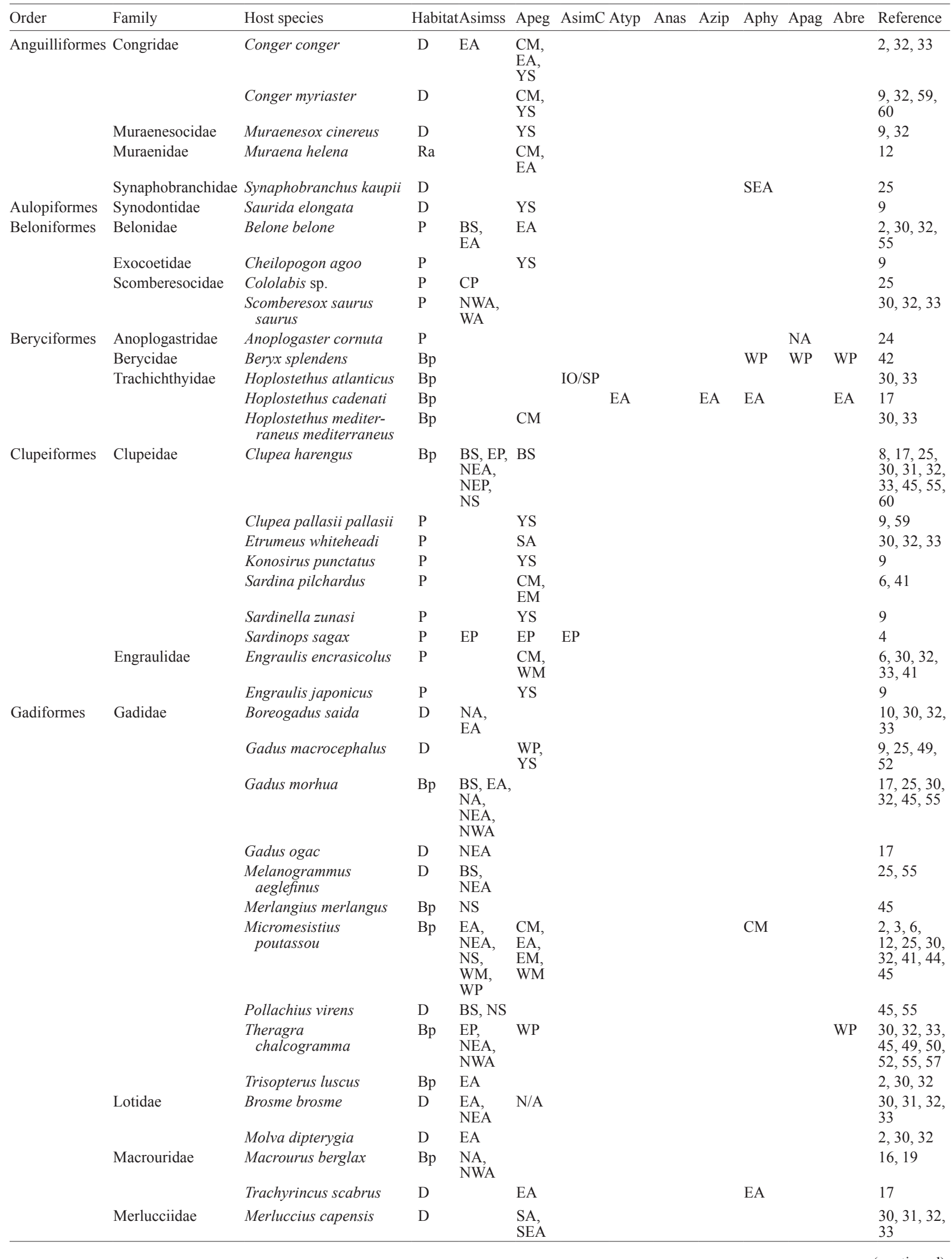


Table 2. Continued.

\begin{tabular}{|c|c|c|c|c|c|c|c|c|c|c|c|c|c|}
\hline Order & Family & Host species & $\begin{array}{l}\text { Habi- } \\
\text { tat }\end{array}$ & Asimss & Apeg & AsimC & Atyp & Anas & Azip & Aphy & Apag & Abre & Reference \\
\hline & & Merluccius hubbsi & $\mathrm{Bp}$ & & SWA & & & & & & & & $\begin{array}{l}30,31,32, \\
33\end{array}$ \\
\hline & & Merluccius merluccius & $\mathrm{D}$ & $\begin{array}{l}\text { BS, } \\
\text { EA, } \\
\text { EM, } \\
\text { NEA, } \\
\text { SM, } \\
\text { WM }\end{array}$ & $\begin{array}{l}\text { CM, } \\
\text { EA, } \\
\text { EM, } \\
\text { NEA, } \\
\text { SM, } \\
\text { WM }\end{array}$ & & $\begin{array}{l}\text { CM, } \\
\text { EA, } \\
\text { EM, } \\
\text { WM }\end{array}$ & EA & $\begin{array}{l}\text { EA, } \\
\text { EM }\end{array}$ & $\begin{array}{l}\text { CM, } \\
\text { EM, } \\
\text { EA, } \\
\text { SM, } \\
\text { WM }\end{array}$ & NEA & $\begin{array}{l}\text { EA, } \\
\text { EM, } \\
\text { SM }\end{array}$ & $\begin{array}{l}2,6,11, \\
12,25,30, \\
31,32,33, \\
34,35,36, \\
37,38,41, \\
45,55\end{array}$ \\
\hline & & Merluccius polli & $\mathrm{D}$ & & EA & & EA & EA & & EA & & & 17 \\
\hline & Moridae & Pseudophycis bachus & $\mathrm{D}$ & & $\mathrm{SP}$ & $\mathrm{SP}$ & & & & & & & $\begin{array}{l}30,31,32, \\
33\end{array}$ \\
\hline & Phycidae & Phycis blennoides & $\mathrm{Bp}$ & & $\mathrm{CM}$ & & & & & $\mathrm{CM}$ & & & 12,32 \\
\hline & & Phycis phycis & $\mathrm{Bp}$ & & $\begin{array}{l}\text { CM, } \\
\text { EA }\end{array}$ & & $\begin{array}{l}\text { CM, } \\
\text { EA }\end{array}$ & & & $\begin{array}{l}\text { CM, } \\
\text { EA }\end{array}$ & & & 12,32 \\
\hline \multirow[t]{3}{*}{ Lophiiformes } & Lophiidae & Lophius litulon & $\mathrm{D}$ & & YS & & & & & & & & 9 \\
\hline & & Lophius piscatorius & $\mathrm{D}$ & $\begin{array}{l}\text { BS, } \\
\text { EA }\end{array}$ & $\begin{array}{l}\text { CM, } \\
\text { EA, } \\
\text { SM }\end{array}$ & & & & & & & & 59 \\
\hline & & Lophius vomerinus & $\mathrm{D}$ & & SA & & & & & & & & $30,32,33$ \\
\hline Mugiliformes & Mugilidae & Mugil cephalus & $\mathrm{Bp}$ & & YS & & & & & & & & 59 \\
\hline \multirow{4}{*}{$\begin{array}{l}\text { Myctophi- } \\
\text { formes }\end{array}$} & Myctophidae & Electrona carlsbergi & $\mathrm{P}$ & & & SA & & & & & & & 32 \\
\hline & & Gymnoscopelus nicholsi & $\mathrm{P}$ & & SA & SA & & & & & & & 22,25 \\
\hline & & Myctophum punctatum & $\mathrm{P}$ & $\mathrm{CA}$ & $\mathrm{CA}$ & & & & & & & & 21,23 \\
\hline & & Notoscopelus kroyeri & $\mathrm{P}$ & $\mathrm{CA}$ & & & & & & & & & 21 \\
\hline Ophidiiformes & Ophidiidae & Genypterus capensis & $\mathrm{D}$ & & SA & & & & & & & & $30,32,33$ \\
\hline \multirow[t]{3}{*}{ Osmeriformes } & Argentinidae & Argentina silus & $\mathrm{D}$ & NA & & & & & & & & & 19 \\
\hline & Bathylagidae & Bathylagus euryops & $\mathrm{P}$ & NA & & & & & & & & & $19,30,32$ \\
\hline & Osmeridae & Hypomesus japonicus & $\mathrm{D}$ & WP & & & & & & & & & 32,57 \\
\hline \multirow[t]{25}{*}{ Perciformes } & Bramidae & Brama brama & $\mathrm{P}$ & & SA & & & & & & & & $30,32,33$ \\
\hline & Caesionidae & Caesio cuning & $\mathrm{Ra}$ & & & & WP & & & & & & 25 \\
\hline & Carangidae & Decapterus maruadsi & $\mathrm{Ra}$ & & YS & & & & & & & & 9 \\
\hline & & Selar crumenophthalmus & $\mathrm{Ra}$ & & & & $\begin{array}{l}\mathrm{CP} \\
\mathrm{WP}\end{array}$ & & & & & & $25,32,60$ \\
\hline & & Seriola dumerili & $\mathrm{Ra}$ & & WP & & & & & & & & 49 \\
\hline & & Seriola lalandi & $\mathrm{Bp}$ & & YS & & & & & & & & 9 \\
\hline & & Trachurus capensis & $\mathrm{P}$ & & SA & & & & & & & & $30,32,33$ \\
\hline & & Trachurus mediterraneus & $\mathrm{P}$ & EA & $\begin{array}{l}\text { CM, } \\
\text { EA }\end{array}$ & & & & & & & & $\begin{array}{l}11,30,32 \\
33,41\end{array}$ \\
\hline & & Trachurus picturatus & $\mathrm{Bp}$ & EA & EA & & EA & & & & & & $\begin{array}{l}30,32,33 \\
35,41,48\end{array}$ \\
\hline & & Trachurus trachurus & $\mathrm{P}$ & $\begin{array}{l}\text { EA, } \\
\text { EM, } \\
\text { CM, } \\
\text { NEA, } \\
\text { NS, } \\
\text { WM }\end{array}$ & $\begin{array}{l}\text { CM, } \\
\text { EA, } \\
\text { EM, } \\
\text { NEA, } \\
\text { SWP, } \\
\text { WM }\end{array}$ & & $\begin{array}{l}\text { EA, } \\
\text { EM }\end{array}$ & EA & & $\mathrm{CM}$ & EA & & $\begin{array}{l}2,3,6,11 \\
17,25,27 \\
30,31,32 \\
33,38,39 \\
41,44,45\end{array}$ \\
\hline & Centracanthidae & Spicara smaris & $\mathrm{D}$ & & EM & & & & & & & & 6 \\
\hline & Coryphaenidae & Coryphaena hippurus & $\mathrm{P}$ & & YS & & $\begin{array}{l}\text { IO, } \\
\text { WP }\end{array}$ & & & & & & $\begin{array}{l}9,32,35 \\
47,59\end{array}$ \\
\hline & Emmelichthyidae & $\begin{array}{l}\text { Emmelichthys nitidus } \\
\text { nitidus }\end{array}$ & $\mathrm{D}$ & & SA & & & & & & & & $30,32,33$ \\
\hline & Gempylidae & Thyrsites atun & $\mathrm{Bp}$ & & $\begin{array}{l}\text { SA, } \\
\text { SEA }\end{array}$ & $\begin{array}{l}\text { SA, } \\
\text { SEA }\end{array}$ & & & & & & & $\begin{array}{l}30,31,32, \\
33\end{array}$ \\
\hline & Lateolabracidae & Lateolabrax japonicus & $\mathrm{Ra}$ & & YS & & & & & & & & 9 \\
\hline & Lutjanidae & Lutjanus erythropterus & $\mathrm{Ra}$ & & YS & & & & & & & & 9 \\
\hline & Moronidae & Dicentrarchus labrax & $\mathrm{D}$ & NEA & & & & & & & & & 5 \\
\hline & Mullidae & Mullus barbatus barbatus & $\mathrm{D}$ & EM & & & & & & & & & 6 \\
\hline & & Mullus surmuletus & $\mathrm{D}$ & & $\mathrm{CM}$ & & & & & & & & 41 \\
\hline & Nemipteridae & Nemipterus bathybius & $\mathrm{D}$ & & & & WP & & & & & & 32,60 \\
\hline & & Nemipterus virgatus & $\mathrm{D}$ & & & & WP & & & & & & 32,60 \\
\hline & Nototheniidae & Notothenia coriiceps & $\mathrm{D}$ & & & SEA & & & & & & & 9 \\
\hline & & Notothenia rossii & $\mathrm{D}$ & & & SEA & & & & & & & 9 \\
\hline & Pholidae & Pholis nebulosa & $\mathrm{D}$ & & YS & & & & & & & & 9 \\
\hline & Pinguipedidae & Parapercis colias & $\mathrm{D}$ & & $\mathrm{SP}$ & $\mathrm{SP}$ & & & & & & & $\begin{array}{l}30,31,32, \\
33\end{array}$ \\
\hline
\end{tabular}

(continued) 
Kuhn et al.: Anisakis spp. in teleosts

Table 2. Continued.

\begin{tabular}{|c|c|c|c|c|c|c|c|c|c|c|c|c|}
\hline Order & Family & Host species & $\begin{array}{l}\text { Habi- } \\
\text { tat }\end{array}$ & Asimss & Apeg & AsimC Atyp & Anas & Azip & Aphy & Apag & Abre & Reference \\
\hline & Rachycentridae & Rachycentron canadum & $\mathrm{Ra}$ & & YS & & & & & & & 9 \\
\hline & Sciaenidae & Collichthys niveatus & $\mathrm{D}$ & & YS & & & & & & & 9 \\
\hline & & Larimichthys polyactis & $\mathrm{Bp}$ & & YS & & & & & & & 9 \\
\hline & & Nibea albiflora & $\mathrm{Bp}$ & & YS & & & & & & & 9 \\
\hline & & Pennahia argentata & $\mathrm{Bp}$ & & YS & & & & & & & 9 \\
\hline & Scombridae & Auxis rochei rochei & $\mathrm{P}$ & & & WP & & & & & & 25,47 \\
\hline & & Auxis thazard thazard & $\mathrm{P}$ & & YS & $\begin{array}{l}\text { WA, } \\
\text { WP, } \\
\text { YS }\end{array}$ & & & WA & & & $\begin{array}{l}9,14,25 \\
35\end{array}$ \\
\hline & & Euthynnus affinis & $\mathrm{P}$ & & & IO & & & & & & 35 \\
\hline & & Euthynnus alletteratus & $\mathrm{Ra}$ & EA & EA & & & & & & & $\begin{array}{l}11,30,32, \\
33,41\end{array}$ \\
\hline & & Katsuwonus pelamis & $P$ & & & $\mathrm{CP}$ & & & & & & 25 \\
\hline & & Scomber australasicus & $\mathrm{P}$ & WP & WP & WP & & & & & & $7,49,52$ \\
\hline & & Scomber colias & $\mathrm{P}$ & & EM & & & & & & & 6,33 \\
\hline & & Scomber japonicus & $\mathrm{P}$ & $\begin{array}{l}\text { EA, } \\
\text { WM, } \\
\text { WP }\end{array}$ & $\begin{array}{l}\text { CM, } \\
\text { EA, } \\
\text { WM, } \\
\text { WP, } \\
\text { YS }\end{array}$ & $\begin{array}{l}\text { EA, } \\
\text { IO }\end{array}$ & EA & EA & $\begin{array}{l}\text { EA, } \\
\text { WM }\end{array}$ & & & $\begin{array}{l}1,3,9,11, \\
12,17,25, \\
26,30,32, \\
45,48,49, \\
56,57,58, \\
59\end{array}$ \\
\hline & & Scomber scombrus & $\mathrm{P}$ & $\begin{array}{l}\text { BS, EA, } \\
\text { EM, } \\
\text { CM, } \\
\text { NEA, } \\
\text { NS, } \\
\text { WM }\end{array}$ & $\begin{array}{l}\text { BS, } \\
\text { CM, } \\
\text { EA, } \\
\text { EM, } \\
\text { NEA, } \\
\text { WM }\end{array}$ & $\begin{array}{l}\text { CM, } \\
\text { EA }\end{array}$ & EA & EA & $\begin{array}{l}\text { CM, } \\
\text { EA }\end{array}$ & & & $\begin{array}{l}2,3,6,11 \\
12,25,30 \\
32,33,41 \\
45,55\end{array}$ \\
\hline & & $\begin{array}{l}\text { Scomberomorus } \\
\text { commerson }\end{array}$ & $\mathrm{P}$ & & & IO & & & & & & 35 \\
\hline & & $\begin{array}{l}\text { Scomberomorus nipho- } \\
\text { nius }\end{array}$ & $\mathrm{P}$ & & YS & & & & & & & $\begin{array}{l}9,25,49 \\
59\end{array}$ \\
\hline & & Thunnus thynnus & $\mathrm{P}$ & WP & $\begin{array}{l}\text { CM, } \\
\text { WA }\end{array}$ & WA & & & & & & $\begin{array}{l}12,30,31 \\
32,33,35\end{array}$ \\
\hline & Sillaginidae & Sillago sihama & $\mathrm{Ra}$ & & YS & & & & & & & 9 \\
\hline & Sparidae & Boops boops & $\mathrm{D}$ & & $\mathrm{CM}$ & & & & & & & 45 \\
\hline & & Diplodus annularis & $\mathrm{Bp}$ & & EM & & & & & & & 6 \\
\hline & & Pagellus bogaraveo & $\mathrm{Bp}$ & EA & EA & EA & & EA & EA & & & 13 \\
\hline & & Spondyliosoma cantharus & $\mathrm{Bp}$ & EA & & & & & & & & 2,30 \\
\hline & Stromateidae & Pampus argenteus & $\mathrm{P}$ & & YS & & & & & & & 9 \\
\hline & Trachinidae & Aphanopus carbo & $\mathrm{Bp}$ & EA & EA & & EA & EA & EA & EA & & $\begin{array}{l}25,37,40, \\
48\end{array}$ \\
\hline & & Echiichthys vipera & $\mathrm{D}$ & & $\mathrm{CM}$ & & & & & & & 12 \\
\hline & & Lepidopus caudatus & $\mathrm{Bp}$ & & $\begin{array}{l}\text { CM, } \\
\text { SEA }\end{array}$ & & & & & & & $\begin{array}{l}30,31,32, \\
33,45\end{array}$ \\
\hline & & Lepturacanthus savala & $\mathrm{Bp}$ & & & WP & & & & & & 25 \\
\hline & & Trachinus draco & $\mathrm{D}$ & & $\mathrm{CM}$ & & & & & & & 12 \\
\hline & & Trichiurus lepturus & $\mathrm{Bp}$ & WP & $\begin{array}{l}\text { CM, } \\
\text { WP, } \\
\text { YS }\end{array}$ & WP & & & & & & $\begin{array}{l}9,12,25 \\
26,54\end{array}$ \\
\hline & Trichodontidae & Arctoscopus japonicus & $\mathrm{D}$ & & WP & & & & & & & 52 \\
\hline & Xiphiidae & Xiphias gladius & $\mathrm{P}$ & $\mathrm{CM}$ & $\begin{array}{l}\text { CM, } \\
\text { EA }\end{array}$ & EA & & $\begin{array}{l}\text { CM, } \\
\text { EA, } \\
\text { WM }\end{array}$ & $\begin{array}{l}\mathrm{CM}, \\
\mathrm{WM}\end{array}$ & $\mathrm{CA}$ & $\begin{array}{l}\text { CA, } \\
\text { EA }\end{array}$ & $30,32,33$ \\
\hline & Zoarcidae & Zoarces elongatus & $\mathrm{D}$ & & YS & & & & & & & 9 \\
\hline \multirow{8}{*}{$\begin{array}{l}\text { Pleuronecti- } \\
\text { formes }\end{array}$} & Bothidae & Arnoglossus imperialis & $\mathrm{D}$ & EA & EA & & & & & & & $28,29,32$ \\
\hline & Citharidae & Citharus linguatula & $\mathrm{D}$ & $\begin{array}{l}\text { EA, } \\
\text { NEA }\end{array}$ & $\begin{array}{l}\text { EA, } \\
\text { NEA }\end{array}$ & & & & & & & $28,29,32$ \\
\hline & Paralichthyidae & Paralichthys olivaceus & $\mathrm{D}$ & EXP & EXP & & & & & & & 51 \\
\hline & Pleuronectidae & Cleisthenes herzensteini & D & & YS & & & & & & & 9,25 \\
\hline & & Hippoglossoides dubius & $\mathrm{D}$ & & WP & & & & & & & 52 \\
\hline & & $\begin{array}{l}\text { Hippoglossus } \\
\text { hippoglossus }\end{array}$ & $\mathrm{D}$ & $\mathrm{BE}$ & & & & & & & & 30,32 \\
\hline & & Platichthys flesus & $\mathrm{D}$ & & & EA & & & & & & $28,29,32$ \\
\hline & & $\begin{array}{l}\text { Pseudopleuronectes } \\
\text { yokohamae }\end{array}$ & $\mathrm{D}$ & & YS & & & & & & & 9 \\
\hline
\end{tabular}


Table 2. Continued.

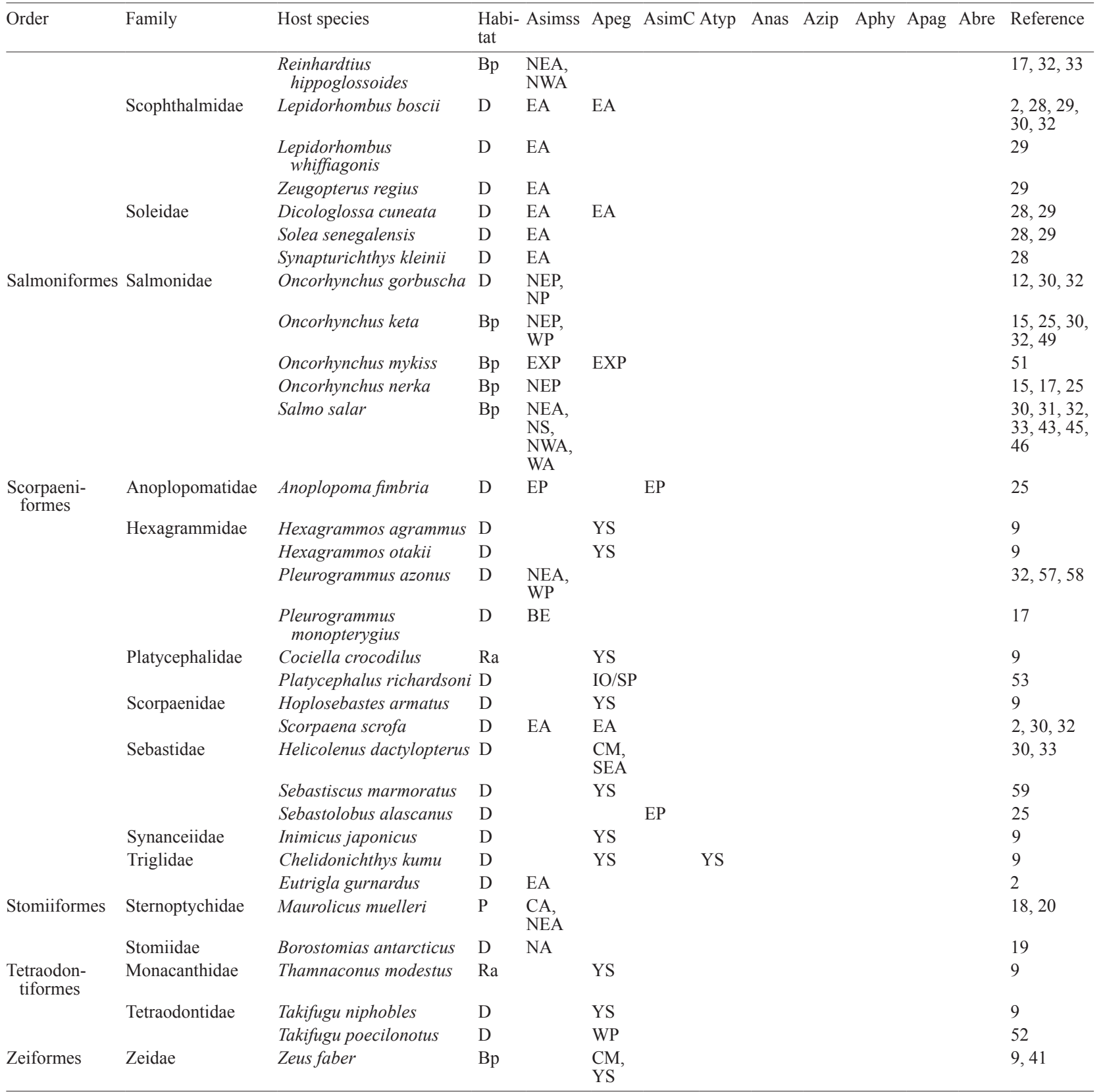

Asimss - A. simplex s.s.; Apeg - A. pegreffi; AsimC - A. simplex C; Atyp - A. typica; Anas - A. nascettii; Azip - A. ziphidarum; Aphy - A. physeteris; Apag - A. paggiae; Abre - A. brevispiculata . Bp - benthopelagic; D - demersal; P - pelagic; Ra - reef-associated. BE - Bering Sea; BS - Baltic Sea; CA - Central Atlantic; CM - Central Mediterranean Sea; CP - Central Pacific; EA - East Atlantic; EM - East Mediterranean Sea; EP - East Pacific; EXP - experimental infection; IO - Indian Ocean; NA - North Atlantic; N/A - Not Available; NEA - North East Atlantic; NEP - North East Pacific; NP - North Pacific; NS - North Sea; NWA - North West Atlantic; SA - South Atlantic; SEA - South East Atlantic; SM - South Mediterranean Sea; SP - South Pacific; SWA - South West Atlantic; SWP - South West Pacific; WA - West Atlantic; WM - West Mediterranean Sea; WP - West Pacific; YS - Yellow Sea. 1 - Abattouy et al. 2011; 2 - Abollo et al. 2001; 3 - Abollo et al. 2003; 4 - Baldwin et al. 2011; 5 - Bernadi et al. 2011; 6 - Chaligiannis et al. 2012; 7 - Chou et al. 2011; 8 - Cross et al. 2007; 9 - Du et al. 2010; 10 - Dzido et al. 2009; 11 - Farjallah et al. 2008a; 12 - Farjallah et al. 2008b; 13 - Hermida et al. 2012; 14 - Iñiguez et al. 2009; 15 - Karl et al. 2011; 16 - Kellermanns et al. $2007 ; 17$ - Kijewska et al. 2009; 18 - Klimpel et al. 2004; 19 - Klimpel et al. 2006; 20 - Klimpel et al. 2007; 21 - Klimpel et al. 2008; 22 - Klimpel et al. 2010a; 23 - Klimpel et al. 2010b; 24 - Klimpel et al. 2011; 25 - Kuhn et al. 2011; 26 - Lee et al. 2009; 27 - MacKenzie et al. 2002; 28 - Marques et al. 2006; 29 - Marques et al. 2009; 30 - Mattiucci and Nascetti 2006; 31 - Mattiucci and Nascetti 2007; 32 - Mattiucci and Nascetti 2008; 33 - Mattiucci et al. 1997; 34 - Mattiucci et al. 2001; 35 - Mattiucci at el. 2002; 36 - Mattiucci et al. 2004; 37 - Mattiucci et al. 2005; 38 - Mattiucci et al. 2007; 39 - Mattiucci et al. 2008; 40 - Mattiucci et al. 2009; 41 - Meloni et al. 2011; 42 - Murata et al. 2011; 43 - Murphy et al. 2010; 44 - Nadler et al. 2005; 45 - Nascetti et al. 1986; 46 - Noguera et al. 2009; 47 - Palm et al. 2008; 48 - Pontes et al. 2005; 49 - Quiazon et al. 2008; 50 - Quiazon et al. 2009; 51 - Quiazon et al. 2011a; 52 - Quiazon et al. 2011b; 53 - Shamsi et al. 2011; 54 - Shih 2004; 55 - Skov et al. 2010; 56 - Suzuki et al. 2010; 57 - Umehara et al. 2006; 58 - Umehara et al. 2008; 59 - Zhang et al. 2007; 60-Zhu et al. 2007. 


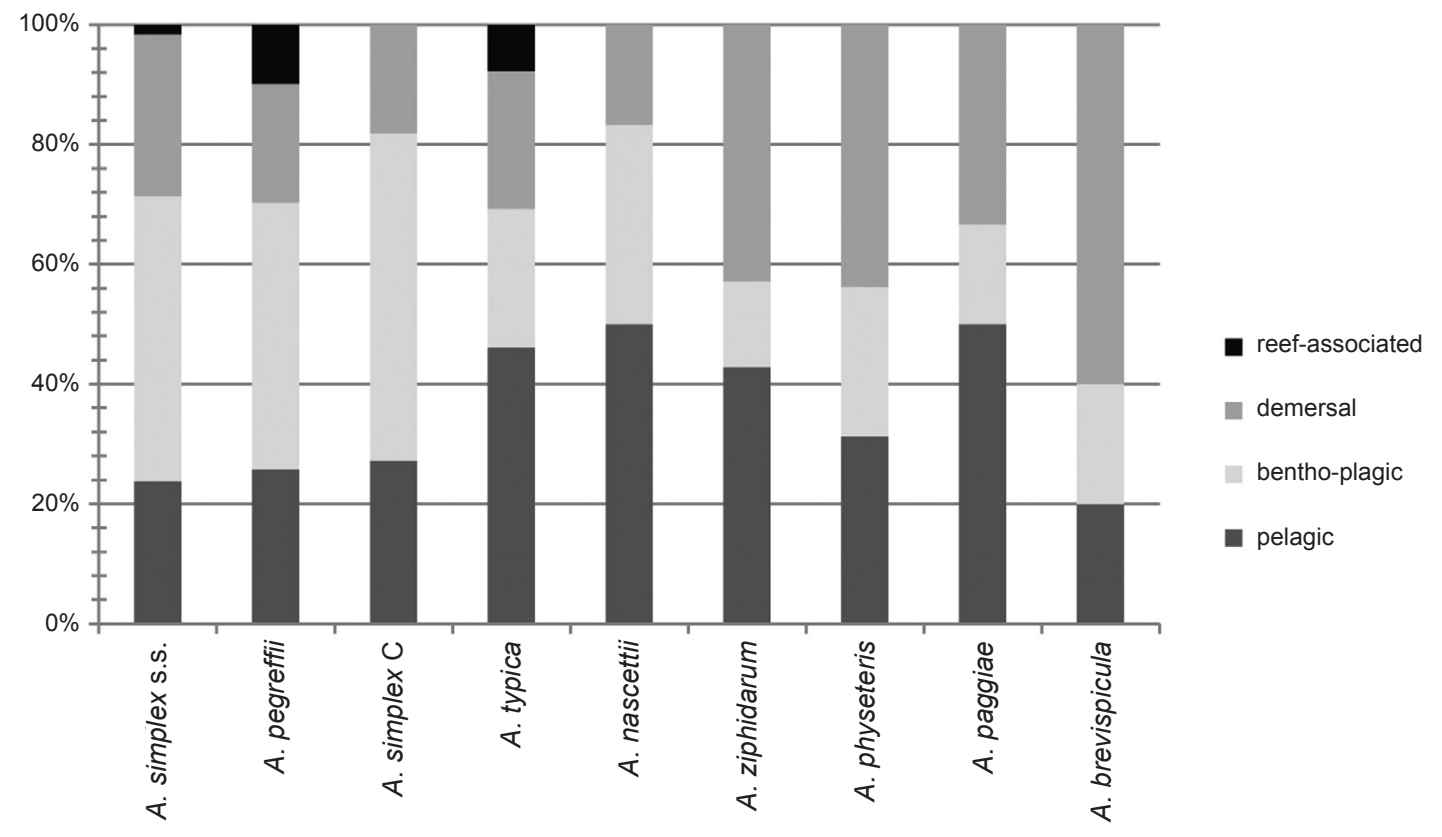

Fig. 1. Proportions of Anisakis teleost fish hosts in demersal, benthopelagic, pelagic and reef-associated habitats.

seter macrocephalus Linnaeus, Mesoplodon grayi von Haast, Kogia breviceps (Blainville) and K. sima (Owen), which are known to feed especially in the pelagic realm, the life cycle of these nematodes has to shift at some point from (bentho-)pelagic habitats into the free water column (Table 2, Fig. 1). Palm et al. (2008) recorded 21 mainly pelagic or reef associated fish species as intermediate hosts for Anisakis spp., four of them for A. typica and two closely related genotypes. Klimpel et al (2011) identified A. paggiae in the common fangtooth Anoplogaster cornuta (Valenciennes) in the Irminger Sea and extended the distribution range of their definitive hosts (Kogia breviceps and $K$. sima) reported so far.

Mesopelagic fishes such as $A$. cornuta transfer parasites into larger paratenic hosts (e.g. Xiphias gladius Linnaeus) and may therefore play a crucial role within the life cycle of this anisakid nematode. Furthermore, the authors suggested similar life cycles for A. paggiae and the other species of the $A$. physeteris species complex (A. brevispiculata, A. physeteris) as well as for both sister species $A$. nascettii and $A$. zipihdarum in meso/bathypelagic waters, in accordance with the most common feeding ecology and depth range of their reported intermediate fish and definitive hosts (Klimpel et al. 2011). The latter four Anisakis species mainly use the same (meso-)pelagic, piscivorous fish species such as Aphanopus carbo Lowe, Beryx splendens Lowe, Hoplostethus cadenati Quéro, Merluccius merluccius and M. polli (Cadenat) as well as Pagellus bogaraveo (Brünnich) in their life cycles (Table 2).

Our data confirm that Anisakis species are less hostspecific regarding the intermediate teleost hosts. Busch et al. (2012) demonstrated that Anisakis larvae can be found in crustaceans from pelagic, benthopelagic and benthic habitats. Euphausiids are considered important crustacean hosts that transmit larvae directly to baleen whales, which feed on them extensively (Marcogliese 1995). By utilizing intermediate hosts from different trophic levels and habitats within the marine food webs, Anisakis larvae are abundant in virtually every depth horizon, in both the pelagic and benthic zone. However, taking into account the distribution, habitats and feeding behavior of toothed- and baleen whales, transmission to their respective definitive hosts has to be associated with the free water column.

Acknowledgments. We thank Simonetta Mattiucci (Department of Health Sciences, Rome), Armand Kuris (University of California, Santa Barbara), Greta Smith Aeby (Hawai'i Institute of Marine Biology) and Horst Karl (Max Rubner Institute, Hamburg) for providing parasite material from the Ionian and Tyrrhenian Sea, Hawaii, California and Alaska, respectively. Material collection from Indonesian waters was done within the framework of the joint Indonesian-German research programme SPICE I (Science for the Protection of Indonesian Coastal Marine Ecosystems, BMBF Grant No. 03F0391A) and under cooperation with I Made Damriyasa, Faculty of Veterinary Medicine, UDAYANA University, Bali (H.W. Palm). We thank Christian Kuhn for programming shell scripts. This study was financially supported by the research funding programme 'LOEWE - Landes-Offensive zur Entwicklung Wissenschaftlich-ökonomischer Exzellenz' of Hesse's Ministry of Higher Education, Research and the Arts, and the German Research Council (DFG KL 2087/1-1, 1-2, DFG PA 664/4-1, 6-1). 
Abattouy N., Valero A., Benajiba M.H., Lozano J., MartínSÁNCHeZ J. 2011: Anisakis simplex s.l. parasitation in mackerel (Scomber japonicus) caught in the north of Morocco - prevalence and analysis of risk factors. Int. J. Food Microbiol. 150: $136-139$.

Abollo E., Gestal C., Pascual S. 2001: Anisakis infestation in marine fish and cephalopods from Galician waters: an updated perspective. Parasitol. Res. 87: 492-499.

Abollo E., Paggi L., Pascual S., D’Amelio S. 2003: Occurrence of recombinant genotypes of Anisakis simplex s.s. and Anisakis pegreffii (Nematoda: Anisakidae) in an area of sympatry. Infect. Genet. Evol. 3: 175-181.

Altschul S.F., Gish W., Myers W.M.E.W., Lipmann D.J. 1990: Basic local alignment search tool. J. Mol. Biol. 215: 403-410.

Anderson R.C. 2000: Nematode Parasites of Vertebrates. Their Development and Transmission. CABI, Wallingford, $650 \mathrm{pp}$.

Anderson T.J.C., Blouin M.S., Beech R.N. 1998: Population biology of parasitic nematodes: applications of genetic markers. Adv. Parasitol. 41: 219-283.

Audicana M.T., Kennedy M.W. 2008: Anisakis simplex: from obscure infectious worm to inducer of immune hypersensitivity. Clin. Microbiol. Rev. 21: 360-379.

BALDWIN R.E., BAnKs M.A., JaCoBson K.C. 2011: Integrating fish and parasite data as a holistic solution for identifying the elusive stock structure of Pacific sardines (Sardinops sagax). Rev. Fish Biol. Fish. 22: 137-156.

Bernadi C., Gustinelli A., Fioravanti M.L., Caffara M., Mattiucci S., Cattaneo P. 2011: Prevalence and mean intensity of Anisakis simplex (sensu stricto) in European sea bass (Dicentrarchus labrax) from Northeast Atlantic Ocean. Int. J. Food Microbiol. 148: 55-59.

Busch M.W., Kuhn T., Münster J., Klimpel S. 2012: Marine crustaceans as potential hosts and vectors for metazoan parasites. In: H. Mehlhorn (Ed.), Arthropods as Vectors of Emerging Diseases. Parasitology Research Monographs. Volume 3, Springer-Verlag, Heidelberg, pp. 329-360.

Chaligiannis I., Lalle M., Pozio E., Sotiraki S. 2012: Anisakidae infection in fish of the Aegean Sea. Vet. Parasitol. 184: 362-366.

Chou Y.-Y., Wang C.-S., Chen H.-G., Chen H.-Y., Chen S.-N., Sнін H.-H. 2011: Parasitism between Anisakis simplex (Nematoda: Anisakidae) third-stage larvae and the spotted mackerel Scomber australasicus with regard to the application of stock identification. Vet. Parasitol. 177: 324-331.

Cross M.A., Collins C., Campbell N., Watts P.C., Chubb J.C., Cunningham C.O., Hatfield E.M.C., MacKenzie K. 2007: Levels of intra-host and temporal sequence variation in a large CO1 sub-units from Anisakis simplex sensu stricto (Rudolphi 1809) (Nematoda: Anisakidae): implications for fisheries management. Mar. Biol. 151: 695-702.

Daschner A., Cuéllar C., Rodero M. 2011: The Anisakis allergy debate: does an evolutionary approach help? Trends Parasitol. 28: 9-15.

Daschner A., Pascual C-Y. 2005: Anisakis simplex: sensitization and clinical allergy. Curr. Opin. Allergy Clin. Immunol. 5: 281-285.

Du C., Zhang L., Shi M., Ming Z., Hu M., Gasser R.B. 2010: Elucidating the identity of Anisakis larvae from a broad range of marine fishes from the Yellow Sea, China, using a combined electrophoretic-sequencing approach. Electrophoresis 31: 654658 .
Dzido J., Kijewska A., Rokicka M., Swiątalska-Koseda A., RокICKI J 2009: Report on anisakid nematodes in polar regions - preliminary results. Polar Sci. 3: 207-211.

Farjallah S., Busi M., Mahjoub M.O., Slimane B.B., Paggi L., Said K., D'Amelio S. 2008a: molecular characterization of larval anisakid nematodes from marine fishes off the Moroccan and Mauritanian coasts. Parasitol. Int. 57: 430-436.

Farjallah S., Slimane B.B., Busi M., Paggi L., Amor N., Blel H., Said K., D’Amelio S. 2008b: Occurrence and molecular identification of Anisakis spp. from the North African coasts of Mediterranean Sea. Parasitol. Res. 102: 371-379.

Froese R., Pauly D. (Eds.) 2012: Fishbase World Wide Web electronic publication, www.fishbase.org, accessed 08/2012.

Hailer F., Kutschera V.e., Hallström B.M., Klassert D., Fain S.R., Leonard J.A., Arnason U., Janke A. 2012: Nuclear genomic sequences reveal that polar bears are an old and distinct bear lineage. Science 336: 344-347.

Hammerschmidt K., Koch K., Milinski M., Сhubb J.C., ParkER G.A. 2009: When to go: optimization of host switching in parasites with complex life cycles. Evolution 63: 1976-1986.

Hermida M., Mota R., Pacheco C.C., Santos C.L., Cruz C., Saraiva A., Tamagnini P. 2012: Infection levels and diversity of anisakid nematodes in blackspot seabream, Pagellus bogaraveo, from Portuguese waters. Parasitol. Res. 110: 1919-1928.

Hochberg N.S., Hamer D.H. 2010: Anisakidosis: perils of the deep. Clin. Infect. Dis. 51: 806-812.

HøJGAARD D.P. 1998: Impact of temperature, salinity and light on hatching of eggs of Anisakis simplex (Nematoda, Anisakidae), isolated by a new method, and some remarks on survival of larvae. Sarsia 83: 21-28.

Iñiguez A.M., Portes Santos C., Paulo Vicente A.C. 2009: Genetic characterization of Anisakis typica and Anisakis physeteris from marine mammals and fish from the Atlantic Ocean off Brazil. Vet. Parasitol. 165: 350-356.

Jefferson T.A., Webber M.A., Pitman R. 2008: Marine Mammals of the World: A Comprehensive Guide to their Identification. Academic Press, London, 573 pp.

Karl H., Baumann F., Ostermeyer U., Kuhn T., Klimpel S. 2011: Anisakis simplex (s.s.) larvae in wild Alaska salmon: no indication of post-mortem migration from viscera into flesh. Dis. Aquat. Org. 94: 201-209.

Kellermanns E., Klimpel S., Palm H.W. 2007: Molecular identification of ascaridoid nematodes from the deep-sea onion-eye grenadier (Macrourus berglax) from the East Greenland Sea. Deep-Sea Res. I. 54: 2194-2202.

Kijewska A., Dzido J., Shukhgalter O., Rokicki J. 2009: Anisakis parasites of fishes caught on the African shelf. J. Parasitol. 95: 639-645.

Klimpel S., Busch M.W., Kuhn T., Rohde A., Palm H.W. 2010a: The Anisakis simplex complex off the South Shetland Islands (Antarctica): endemic populations versus introduction through migratory hosts. Mar. Ecol-Prog. Ser. 403: 1-11.

Klimpel S., Busch M.W., Sutton T., Palm H.W. 2010b: Mesoand bathy-pelagic fish parasites at the Mid-Atlantic Ridge (MAR): low host specificity and restricted parasite diversity. Deep-Sea Res. I: 57: 596-603.

Klimpel S., Kellermanns E., Palm H.W. 2008: The role of pelagic swarm fish (Myctophidae: Teleostei) in the oceanic lifecycle of Anisakis sibling species at the Mid-Atlantic Ridge, Central Atlantic. Parasitol. Res. 104: 43-53. 
Klimpel S., Kellermanns E., Palm H.W., Moravec F. 2007: Zoogeography of fish parasites of the pearlside (Maurolicus muelleri), with genetic evidence of Anisakis simplex (s.s.) from the Mid-Atlantic Ridge. Mar. Biol. 152: 725-732.

Klimpel S., Kuhn T., Busch M.W., Karl H., Palm H.W. 2011: Deep-water life cycle of Anisakis paggiae (Nematoda: Anisakidae) in the Irminger Sea indicates kogiid whale distribution in north Atlantic waters. Polar Biol. 34: 899-906.

Klimpel S., Palm H.W. 2011: Anisakid nematode (Ascaridoidea) life cycles and distribution: increasing zoonotic potential in the time of climate change? In: H. Mehlhorn (Ed.), Progress in Parasitology. Parasitol. Res. Monographs. Volume 2, SpringerVerlag, Heidelberg, pp. 201-222.

Klimpel S., Palm H.W., Busch M.W., Kellermanns E., RückERT S. 2006: Fish parasites in the Arctic deep-sea: Poor diversity on pelagic fish species vs. heavy parasite load in a demersal fish. Deep-Sea Res. I 53: 1167-1181.

Klimpel S., Palm H.W., Rückert S., Piatkowski U. 2004: The life cycle of Anisakis simplex in the Norwegian Deep (northern North Sea). Parasitol. Res. 94: 1-9.

KøIE M. 2001: Experimental infections of copepods and sticklebacks Gasterosteus aculeatus with small ensheathed and large third-stage larvae of Anisakis simplex (Nematoda, Ascaridoidea, Anisakidae). Parasitol. Res. 87: 32-36.

Køie M., Berland B., Burt M.D.B. 1995: Development to thirdstage larvae occurs in the eggs of Anisakis simplex and Pseudoterranova decipiens (Nematoda, Ascaridoidea, Anisakidae). Can. J. Aquat. Sci. Vol. 52: 134-139.

Kuhn T., García-Màrquez J., Klimpel S. 2011: Adaptive radiation within marine anisakid nematodes: a zoogeographical modeling of cosmopolitan, zoonotic parasites. PLoS ONE 6: e28642.

Larkin M.A., Blackshields G., Brown N.P., Chenna R., McGettingan P.A., McWilliam H., Valentin F., Wallace I.M., Wilm A., Lopez R., Thompson J.D., Gibson T.J., HigGINS D.G. 2007: ClustalW and ClustalX. Bioinformatics 23: 2947-2948.

Lee M.H., Cheon D-S., Chог C. 2009: Molecular genotyping of Anisakis species from Korean Sea fish by polymerase chain reaction-restriction fragment length polymorphism (PCRRFLP). Food Control 20: 623-626.

LiLE N.K. 1998: Alimentary tract helminths of four pleuronectid flatfish in relation to host phylogeny and ecology. J. Fish Biol. 53: 945-953.

MacKenzie K., Campbell N., Mattiucci S., Ramos P., Pinto A.L., Abaunza P. 2002: Parasites as biological tags for stock identification of Atlantic horse mackerel Trachurus trachurus L. Fish. Res. 89: 136-145.

Marcogliese D.J. 1995: The role of zooplankton in the transmission of helminth parasites to fish. Rev. Fish Biol. Fish. 5: 336-371.

Marques J.F., Cabral H.N., Busi M., D’Amelio S. 2006: Molecular identification of Anisakis species from Pleuronectiformes off the Portuguese coast. J. Helminthol. 80: 47-51.

Marques J.F., Santos M.J., Cabral H.N. 2009: Zoogeographical patterns of flatfish (Pleuronectiformes) parasites in the northeast Atlantic and the importance of the Portuguese coast as a transitional area. Sci. Mar. 73: 461-471.

Mattiucci S., Abaunza P., Damiano S., Garcia A., Santos M.N., NAscetti G. 2007: Distribution of Anisakis larvae, identified by genetic markers, and their use for stock characterization of demersal and pelagic fish from European waters: an update. J. Helminthol. 81: 117-127.
Mattiucci S., Abaunza P., Ramadori L., Nascetti G. 2004: Genetic identification of Anisakis larvae in European hake from Atlantic and Mediterranean waters for stock recognition. J. Fish Biol. 65: 495-510.

Mattiucci S., Farina V., Campbell N., MacKenzie K., Ramos P., Pinto A.L., Abaunza P., Nascetti G. 2008: Anisakis spp. larvae (Nematoda: Anisakidae) from Atlantic horse mackerel: their identification and use as biological tags for host stock characterization. Fish. Res. 89: 146-151.

Mattiucci S., Nascetti G. 2006: Molecular systematics, phylogeny and ecology of anisakid nematodes of the genus Anisakis Dujardin, 1845: an update. Parasite 13: 99-113.

Mattiucci S., Nascetti G. 2007: Genetic diversity and infection levels of anisakid nematodes parasitic in fish and marine mammals from boreal and austral hemispheres. Vet. Parasitol. 148: 43-57.

Mattiucci S., Nascetti G. 2008: Advances and trends in the molecular systematics of anisakid nematodes, with implications for their evolutionary ecology and host-parasite co-evolutionary processes. Adv. Parasitol. 66: 47-148.

Mattiucci S., Nascetti G., Cianchi R., Paggi L., Arduino P., Margolis L., Brattey J., Webb S., D’Amelio S., OrecChia P., Bullini L. 1997: Genetic and ecological data on the Anisakis simplex complex with evidence for a new species (Nematoda, Ascaridoidea, Anisakidae). J. Parasitol. 83: 401416.

Mattiucci S., Nascetti G., Dailey M., Webb. S.C., Barros N.B., Cianchi R., Bullini L. 2005: Evidence for a new species of Anisakis Dujardin, 1845: morphological description and genetic relationships between congeners (Nematoda: Anisakidae). Syst. Parasitol. 61: 157-171.

Mattiucci S., Paggi L., Nascetti G., Abollo E., Webb S.C., Pascual S., Cianchi R., Bullini L. 2001: Genetic divergence and reproductive isolation between Anisakis brevispiculata and Anisakis physeteris (Nematoda: Anisakidae). Int. J. Parasitol. 31: 9-14.

Mattiucci S., Paggi L., Nascetti G., Portes Santos C., Costa G., Di Beneditto A.P., Ramos R., Argyrou M., Cianchi R., BULLINI L. 2002: Genetic markers in the study of Anisakis typica (Diesing, 1860): larval identification and genetic relationships with other species of Anisakis Dujardin, 1845 (Nematoda: Anisakidae). Syst. Parasitol. 51: 159-170.

Mattiucci S., Paoletti M., Webi S.C. 2009: Anisakis nascettii n. sp. (Nematoda: Anisakidae) from beaked whales of the southern hemisphere: morphological description, genetic relationships between congeners and ecological data. Syst. Parasitol. 74: 199-217.

McClelland G. 2005: Nematoda (roundworms). In: Rohde K. (Ed.), Marine Parasitology. CABI, Wallingford, pp. 104-115.

Meloni M., Angelucci G., Merella P., Siddi R., Deiana C., Orrù G., Salati F. 2011: Molecular characterization of Anisakis larvae from fish caught off Sardinia. J. Parasitol. 97: 908-914.

Murata R., Suziki J., Sadamasu K., Kai A. 2011: Morphological and molecular characterization of Anisakis larvae (Nematoda: Anisakidae) in Beryx splendens from Japanese waters. Parasitol. Int. 60: 193-198.

Murphy T.M., Berzano M., O’Keeffe S.M., Cotter D.M., McEvoy S.E., Thomas K.A., Maolléidigh N.P.Ó., Whelan K.F. 2010: Anisakid larvae in Atlantic salmon (Salmo salar L.) grilse and post-smolts: molecular identification and histopathology. J. Parasitol. 96: 77-82. 
Nadler S.A., D’Amelio S., Dailey M.D., Paggi L., Siu S., SAKANARI J.A. 2005: Molecular phylogenetics and diagnosis of Anisakis, Pseudoterranova, and Contracaecum from Northern Pacific marine mammals. J. Parasitol. 91: 1413-1429.

Nascetti G., Paggi L., Orecchia P., Smith J.W., Mattiucci S., Bullini L. 1986: Electrophoretic studies on the Anisakis simplex complex (Ascaridida: Anisakidae) from the Mediterranean and north-east Atlantic. Int. J. Parasitol. 16: 633-640.

Noguera P., Collins C., Brno D., Pert C., Turnbull A., McIntosh A., Lester K., Bricknell I., Wallace S., Cook P. 2009: Red vent syndrome in wild Atlantic salmon Salmo salar in Scotland is associated with Anisakis simplex sensu stricto (Nematoda: Anisakidae). Dis. Aquat. Org. 87: 199-215.

Palm H.W., Damriyasa I.M., Linda L., OKa I.B.M. 2008: Molecular genotyping of Anisakis Dujardin, 1845 (Nematoda: Ascaridoidea: Anisakidae) larvae from marine fish of Balinese and Javanese waters, Indonesia. Helminthologia 45: 3-12.

Pontes T., D’Amelio S., Costa G., Paggi L. 2005: Molecular characterization of larval nematodes from marine fishes of Madeira by a PCR-based approach, with evidence for a new species. J. Parasitol. 91: 1430-1434.

Quiazon K.M.A., Yoshinaga T., Ogawa K. 2011a: Experimental challenge of Anisakis simplex sensu stricto and Anisakis pegreffii (Nematoda: Anisakidae) in rainbow trout and olive flounder. Parasitol. Int. 60: 126-131.

Quiazon K.M.A., Yoshinaga T., Ogawa K. 2011b: Distribution of Anisakis species larvae from fishes of the Japanese waters. Parasitol. Int. 60: 223-226.

Quiazon K.M.A., Yoshinaga T., Ogawa K., Yukami R. 2008: Morphological differences between larvae and in vitro-cultured adults of Anisakis simplex (sensu stricto) and Anisakis pegreffii (Nematoda: Anisakidae). Parasitol. Int. 57: 483-489.

Quiazon K.M.A., Yoshinaga T., Santos M.D., Ogawa K. 2009: Identification of larval Anisakis spp. (Nematoda: Anisakidae) in Alaska pollock (Theragra chalcogramma) in northern Japan using morphological and molecular markers. J. Parasitol. 95: $1227-1232$.

Sakanari J.A., McKerrow J.H. 1989: Anisakiasis. Clin. Microbiol. Rev. 2: 278-284.

Shamsi S., Eisenbarth A., Saptarshi S., Beveridge I., GasSER R.B., Lopata A.L. 2011: Occurrence and abundance of

Received 17 September 2012 anisakid nematode larvae in five species of fish from southern Australian waters. Parasitol. Res. 108: 927-934.

Sнiн H.H. 2004: Parasitic helminth fauna of the cutlass fish, Trichiurus lepturus L., and the differentiation of four anisakid nematode third-stage larvae by nuclear ribosomal DNA sequences. Parasitol. Res. 93: 188-195.

Skov J., Kania P.W., Olsen M.M., Lauridsen J.H., BuchMANN K. 2010: Nematode infections of maricultured and wild fishes in Danish waters. Aquaculture 298: 24-28.

Suzuki J., Murata R., Hosaka M., Araki J. 2010: Risk factors for human Anisakis infection and association between the geographic origins of Scomber japonicus and anisakid nematodes. Int. J. Food Microbiol. 137: 88-93.

Umehara A., Kawakami Y., Araki J., Uchida A. 2008: Multiplex PCR for the identification of Anisakis simplex sensu stricto, Anisakis pegreffii and the other anisakid nematodes. Parasitol. Int. 57: 49-53.

Umehara A., Kawakami Y., Matsui T., Araki J., Uchida A. 2006: Molecular identification of Anisakis simplex sensu stricto and Anisakis pegreffii (Nematoda: Anisakidae) from fish and cetacean in Japanese waters. Parasitol. Int. 55: 267-271.

Valentini A., Mattiucci S., Bondanelli P., Webb S.C., Mignucci-Giannone A.A., Colón-Llavina M.M., Nascetti G. 2006: Genetic relationships among Anisakis species (Nematoda: Anisakidae) inferred from mitochondrial cox2 sequences, and comparison with allozyme data. J. Parasitol. 92: 156-166.

Zhang L., Hu M., Shamsi S., Beveridge I., Li H., Xu Z., Li L., Cantacessi C., Gasser R.B. 2007: The specific identification of anisakid larvae from fishes from the Yellow Sea, China, using mutation scanning-coupled sequence analysis of nuclear ribosomal DNA. Mol. Cell. Probe 21: 386-390.

Zhu X.Q., Gasser R.B., Jacobs D.E., Hung G.C., Chilton N.B. 2000: Relationships among some ascaridoid nematodes based on ribosomal DNA sequence data. Parasitol. Res. 86: 738-744.

Zhu X.Q., Podolska M., Liu J.S., Yu H.Q., Chen H.H., Lin Z.X., Luo C.B., Song H.Q., Lin R.Q. 2007: Identification of anisakid nematodes with zoonotic potential from Europe and China by single-strand conformation polymorphism analysis of nuclear ribosomal DNA. Parasitol. Res. 101: 1703-1707.

Accepted 19 December 2012 